\title{
DESIGN, MODELLING AND SIMULATION OF A REMOTELY OPERATED VEHICLE - PART 2
}

\author{
KHOA DUY LE, HUNG DUC NGUYEN, DEV RANMUTHUGALA
}

University of Tasmania / Australian Maritime College; kdle amc.edu.au

Tóm tắt. Nối tiếp theo phần một đã được xuất bản, bài báo tập trung vào việc nâng cấp phần cứng và xây dựng mô hình thực tế ảo cho thiết bị lặn ba động cơ đẩy. Đầu tiên, hệ thống điện tử bao gồm cảm biến và các mạch giao tiếp được thiết lập cho thiết bị lặn. Bộ điều khiển vòng kín sử dụng cấu trúc master-slave bao gồm một máy tính trạm và bộ vi xử lý nguồn mở. Để nâng cao khả năng điều khiển của hệ thống, mô hình thực tế ảo được xây dựng và mô tả các trạng thái của ROV. Dựa vào các tín hiệu phản hồi từ cảm biến, mô hình ảo vận hành tương tự như phương tiện thật. Do đó, nó tăng khả năng giám sát quá trình vận hành ROV trong môi trường mà tầm quan sát bị hạn chế. Cuối cùng, chương trình mô phỏng theo thời gian thực được tiến hành để đánh giá sự tương tác giữa người điều khiển và mô hình ảo. Để hiện thực hóa cảm giác trung thực khi điều khiển, ảnh hưởng của nhiễu từ cảm biến và của dòng chảy được them vào chương trình mô phỏng. Kết quả mô phỏng cho thấy, đáp ứng của thiết bị lặn bền vững bất chấp sự có mặt của nhiễu từ môi trường bên ngoài.

Từ khóa. Phương tiện ngầm, phần cứng nguồn mở, mô hình thực tế ảo.

Abstract. Continuing the previously published study [4], this paper focuses on hardware and Virtual Reality (VR) model development of a three-thruster Remotely Operated Vehicle (ROV). The paper included setting up an on-board electronic system with the associated suite of sensors and the required communication protocol. This system utilises a master-slave structure, which consists of an onshore station computer and an on-board open source microcontroller. To improve the controllability of the driving system, a VR model of the ROV is designed to reflect the altitude and attitude of the physical vehicle. By using the feedback signals from the sensors, the VR model operates in a similar manner to the actual vehicle. Hence, it provides the operator with the capability to monitor the ROV operation within a virtual environment and enables the operator to control the ROV based on the visual inputs and feedback. Finally, real time simulations are presented to validate the interaction between the ROV operator and the VR model. To provide realistic operational conditions, the effects of sensor noise and water current disturbances are included to the simulation programme. The results show that the performance of the VR ROV is stable even with these disturbances.

Key words. Underwater vehicle, open source hardware, virtual reality model.

\section{Nomenclature}

$\begin{array}{ll}\text { Symbol } & \text { Description } \\ J_{o} & \text { Advance ratio } \\ w_{\text {noise }}, v_{\text {noise }} & \text { Process and observation model noise } \\ Q_{\text {noise }}, R_{\text {noise }} & \text { Noise covariance } \\ P & \text { Covariance matrix of error } \\ K & \text { Kalman gain }\end{array}$




$\begin{array}{lll}\text { Symbol } & \text { Unit } & \text { Description } \\ I & \mathrm{kgm}^{2} & \begin{array}{l}\text { moment of inertia of rotational shaft } \\ K_{t}\end{array} \\ K_{b} & \text { N.m/A } & \text { torque constant } \\ Q & \text { N.m } & \text { electromotive force constant } \\ \text { b } & \text { Nms/rad } & \text { Viscous friction coefficient } \\ R_{a} & \Omega & \text { Resistance } \\ L_{a} & \mathrm{H} & \text { Inductance } \\ i_{a} & \mathrm{~A} & \text { Armature current } \\ \omega & \mathrm{Rad} / \mathrm{s} & \text { Angular velocity of the thrusters } \\ V_{a} & \mathrm{~V} & \text { Armature voltage } \\ K_{T} K_{Q} & & \text { Torque and thrust coefficient }\end{array}$

\begin{abstract}
Abbreviation
CFD: Computational Fluid Dynamics, DOF: Degree of Freedom, ROV: Remotely Operated Underwater Vehicle, VR: Virtual Reality, UUV: Unmanned Underwater Vehicles.
\end{abstract}

\title{
1. INTRODUCTION
}

Remotely Operated Vehicles (ROVs) used in the maritime industry are Unmanned Underwater Vehicles (UUVs) that are controlled by human input and via signal transmission cables, from control stations that are remote to the vehicle. Currently, ROVs are used in the maritime industry for a diverse range of functions, including seabed and subsea exploration, underwater inspections, maintenance operations, security tasks, and defence activities. These ROVs are able to replace humans to carry out missions in hostile and hazardous underwater environments. However, controlling ROVs is not a straightforward task due to the highly nonlinear characteristics of the vehicles and external disturbances from the environment such as water current, waves, temperature, and pressure that will influence the performance of the vehicle. In the past, a number of algorithms have been proposed by researchers to meet the control requirements with some well-known examples given below.

Smallwood \& Whitcom [1] have proposed a combination between linear Proportional Derivative (PD) control and adaptive control for a six degree-of-freedom (6-DOF) ROV. Besides linear approaches, intelligent control has also been widely applied to UUVs. For examples, Marzbanrad et al. [2] studied the robust adaptive fuzzy sliding model for trajectory tracking, while Ken et al. [3] implemented fuzzy to develop a docking guidance system for an ROV operating in ocean currents.

In this project, the ROV system described in [4] is modified and upgraded. The sensors systems including the Inertial Measurement Unit (IMU), magnetometer, pressure sensor, etc., are installed on the ROV frame to acquire the states of the vehicle. The sensor data is collected by an Arduino board, a low cost open sources on-board electronic system. The low cost system can be developed on a personal computer or laptop using readily available peripheral devices such as a serial communication board and a microcontroller, thus easily lending itself for undergraduate student projects.

In order to improve the controllability of the driving system, a Virtual Reality (VR) model of the ROV was developed to simulate the behaviour of the vehicleto the different control algorithms $[5,6]$. Based on the feedback signal from the sensor system, the VR model operates exactly in a similar manner to that of the actual vehicle. To validate the interaction between 
operators and the VR model, real time simulations are carried out using the relevant mathematical models. The 6-DOF vehicle model is developed using the appropriate kinetics, which included hydrodynamic and inertia coefficients obtained using a combination of experimental, analytical, and Computational Fluid Dynamics (CFD). In order to provide the effects of a typical marine environment, sensor noise and water currents are added to the simulation programme. Kalman filters and closed-loop control algorithms are utilised within the simulation to improve the controllability of the driving system.

\section{ROV SYSTEM UPGRADE}

\subsection{Control Hardware}

The ROV, namely AMC-ROV-IV [4] shown in Figure 1, is developed as a test vehicle for this project. It consists of a frame constructed from PVC pipes and aluminium with three waterproof dc motor driven propellers, each having a maximum thrust force of $8 \mathrm{~N}$, providing two propulsion thrusters and one vertical thruster.

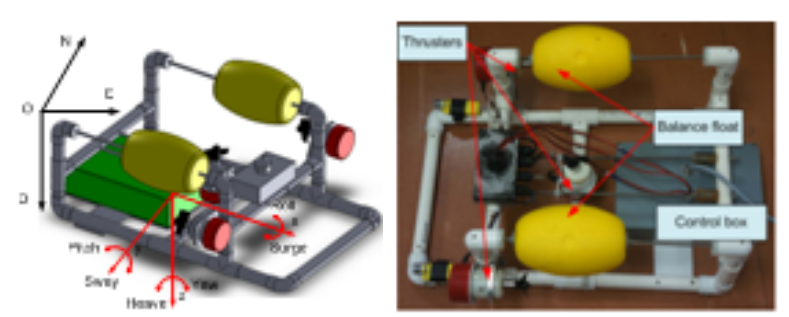

(a) Reference frames

(b) Actual ROV

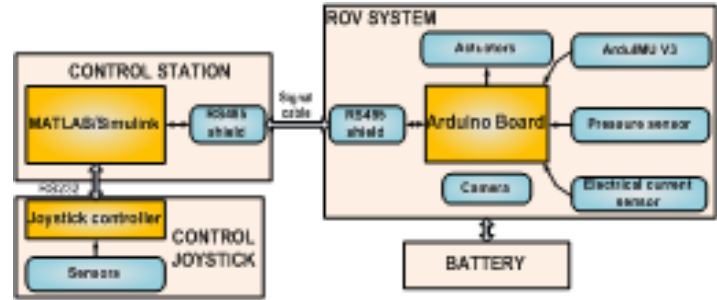

(c) Structure of ROV control system

Figure 1. AMC ROV-IV system and control structure

The control structure of the ROV is shown in Figure 1, consisting of 3 main parts: ROV controller (on-board system), control station (onshore system) and joystick controller.

The operations of the first part are governed by the main Arduino Mega 2560 board. This microcontroller board is connected with the peripheral sensors such as an IMU, digital magnetometer and pressure sensor, which provide the states of the vehicle including acceleration, rotational rate, depth, and direction. All information from the sensors is gathered by the Arduino microcontroller and sent to the control station via a RS-485 serial communication device at the baud rate of $115200 \mathrm{bps}$. The main control algorithm within the station computer receives and processes the raw data, combining with the driving commands from the joystick togenerate control signals to be sent back to the microcontroller via the transmission cable to activate the relevant thrusters. Thus, the microcontroller is required to have only one fixed program to carry out the mission, while the control algorithms, which require higher computational power, are developed and reside within the onshore computer.

The main advantage of the master-slave control structure is the flexibility. It is easier to modify the control algorithm in the station computer than to re-program the microcontroller (on-board system)inside the ROV. In addition, the proposed control structure can be considered as a low cost solution for ROV control, as it does not require any special devices such as embedded computers with high standard I/O interface cards. Complex algorithms can be developed within the onshore computer.

The resolution of the gyroscope and accelerometer within the IMU can be defined by 
modifying the value in the registers of the microprocessor. The measureable range and the resolution of the sensors on the AMC ROV-IV used in this project are given in Table 1.

Table1. Sensors of the ROV

\begin{tabular}{|l|l|l|}
\hline Sensor & Measureable range & Resolution \\
\hline Gyroscope & $\pm 250 \% \mathrm{~s}$ & $16 \mathrm{bit}$ \\
\hline Accelerometer & $\pm 2 \mathrm{~g}$ & $16 \mathrm{bit}$ \\
\hline Magnetometer & \pm 1.3 & $12 \mathrm{bit}$ \\
\hline Pressure sensor & 0 to $75 \mathrm{psi}$ & $10 \mathrm{bit}$ \\
\hline
\end{tabular}

\subsection{ROV and thruster modelling}

In order to verify the control algorithm effects of the external disturbances due to the ocean currents are considered. The velocity vector of the irrotational currents is defined as $\mathrm{v}_{c}=\left[u_{c}, v_{c}, w_{c}, 0,0,0\right]$ with the assumption that the vertical disturbances are neglected. The kinecticequation of the ROV including the current disturbance in [7] can be re-written as [1],

$$
M \dot{v}_{r}+C\left(v_{r}\right) v r+D\left(v_{r}\right) v_{r}+G(\eta)=T,
$$

where $M, C, D, G$ and $T$ are the inertial, coriolis, damping, restoring force and thrust matrices, respectively, and $v_{r}$ defined as $v_{r}=v-v_{c}$ is the relative velocity vector. The details of these matrices can be referred to [5].

In AMC ROV-IV, the three thrusters consist of dc motors connected directly to propellers. Since the speed of an armature controlled dc motor depends on the armature voltage $V_{a}$, the differential equations of a dc motor are given by,

$$
\frac{d}{d t}\left[\begin{array}{c}
\omega \\
i_{a}
\end{array}\right]=\left[\begin{array}{cc}
-\frac{b}{I} & \frac{K_{t}}{I} \\
-\frac{R_{a}}{L_{a}} & -\frac{K_{b}}{L_{a}}
\end{array}\right]\left[\begin{array}{c}
\omega \\
i_{a}
\end{array}\right]+\left[\begin{array}{c}
0 \\
\frac{1}{L_{a}}
\end{array}\right] V_{a}+\left[\begin{array}{c}
-\frac{1}{I} \\
0
\end{array}\right] Q .
$$

The parameters in Equation (2) are defined in Nomenclature.

Based on the rotational speed of the motor shaft and the relative speed of the ROV, the advance ratio $J_{0}$ for the ROV is given by,

$$
J_{o}=\frac{u_{r}}{\rho D \omega|\omega|} .
$$

where $u_{r}, D$ and $\rho$ are surge velocity, propeller diameter and fluid density, respectively.

Fossen [7] showed that the thrust $K_{T}$ and torque $K_{Q}$ coefficients are linear to $J_{0}$. Thus, these coefficients are calculated using the formula

$$
K_{T}=\alpha_{1} J_{o}+\alpha_{2} ; K_{Q}=\beta_{1} J_{o}+\beta_{2},
$$

where $\alpha_{i}$ and $\beta_{i}(i=1,2)$ are four non-dimensional constants, which are determined by the experiments. Next, thrust $T$ and torque $Q$ are calculated by the rotational speed of the motor shaft as

$$
T=\rho D^{4} K_{T}\left(J_{o}\right) \omega|\omega| ; \quad Q=\rho D^{5} K_{Q}\left(J_{o}\right) \omega|\omega|,
$$


where $Q$ is a propeller torque generated by the dc motor described in Equation (2).

\subsection{Re-estimatingthe hydrodynamic coefficients}

Due to the modification of the ROV frame, the CFD analysisand added mass calculation are conducted to re-estimate the coefficients of the system. Thus the coefficients in Part 1 [4] are modified as shown in Table 2 .

Table 2. Estimated ROV coefficients

\begin{tabular}{|c|c|c|c|c|c|c|c|}
\hline Coef & Value & Coef & Value & Coef & Value & Coef & Value \\
\hline$L$ & $480 \mathrm{~mm}$ & $b$ & $290 \mathrm{~mm}$ & $Y_{\dot{v}}$ & $-2.322 \mathrm{~kg}$ & $Y_{v|v|}$ & $-19.37 \mathrm{kgm}^{-1}$ \\
\hline$m$ & $3.2 \mathrm{~kg}$ & $I_{x}$ & $0.091 \mathrm{kgm}^{2}$ & $Z_{\dot{w}}$ & $-2.56 \mathrm{~kg}$ & $Z_{w|w|}$ & $-24.6 \mathrm{kgm}^{-1}$ \\
\hline$I_{y}$ & $0.153 \mathrm{kgm}^{2}$ & $z$ & $75 \mathrm{~mm}$ & $K_{\dot{p}}$ & $-0.045 \mathrm{kgm}^{2}$ & $K_{p|p|}$ & $-0.081 \mathrm{kgm}$ \\
\hline$B$ & $32.5 \mathrm{~N}$ & $l_{1}$ & $0 \mathrm{~mm}$ & $M_{\dot{q}}$ & $-0.068 \mathrm{kgm}^{2}$ & $M_{q|q|}$ & $-0.26 \mathrm{kgm}$ \\
\hline$l_{2}$ & $50 \mathrm{~mm}$ & $l_{3}$ & $180 \mathrm{~mm}$ & $N_{\dot{r}}$ & $0.038 \mathrm{kgm}^{2}$ & $N_{r|r|}$ & $-0.198 \mathrm{kgm}$ \\
\hline$x_{b}$ & $0 \mathrm{~mm}$ & $y_{b}$ & $0 \mathrm{~mm}$ & $X_{u}$ & $-0.65 \mathrm{kgs}^{-1}$ & $K_{p}$ & $-0.029 \mathrm{kgms}^{-1}$ \\
\hline$z_{b}$ & $0.07 \mathrm{~m}$ & $K$ & $0.373 \mathrm{Nm} / \mathrm{V}$ & $Y_{v}$ & $-0.73 \mathrm{kgs}^{-1}$ & $M_{q}$ & $-0.075 \mathrm{kgms}^{-1}$ \\
\hline$X_{\dot{u}}$ & $-1.536 \mathrm{~kg}$ & $X_{u|u|}$ & $-12.6 \mathrm{kgm}^{-1}$ & $Z_{w}$ & $-0.75 \mathrm{kgs}^{-1}$ & $N_{r}$ & $-0.052 \mathrm{kgms}^{-1}$ \\
\hline
\end{tabular}

\section{CONTROL STRUCTURE AND ROV STATES OBSERVATION}

\subsection{Control structure}

In Section 2, the complete dynamic model of the ROV was studied with the voltages to the thruster motors as inputs and the ROV performance as outputs. This section introduces a control algorithm for trajectory tracking, which is defined by the waypoints summarised in Figure 2 .

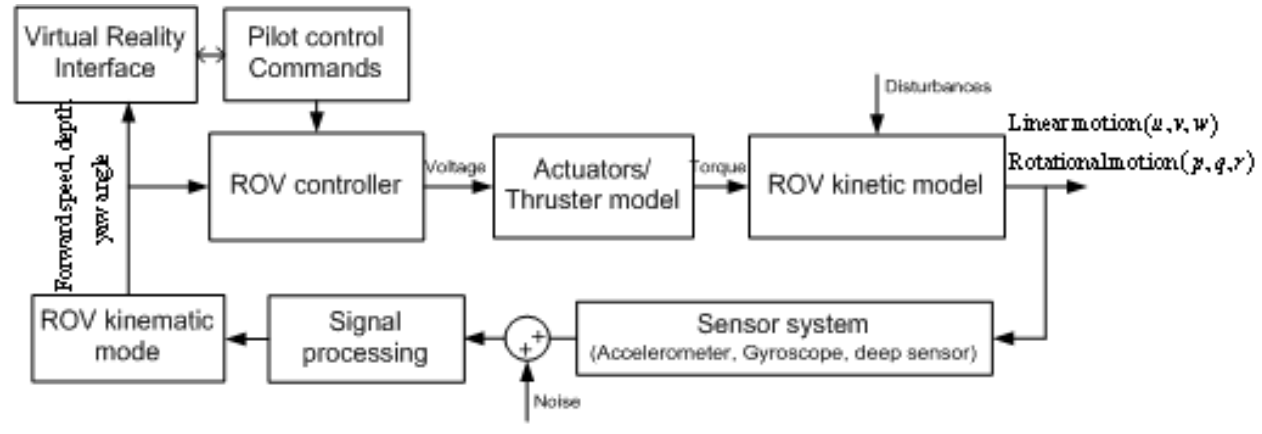

Figure 2. Control diagram of ROV system 
The closed-loop control system consists of the ROV model, the controller, and signal processing. The ROV operator gives the commands to the system via the multi-DOF joystick. The controller synthesises the operator's control commands and the feedback data to generate the appropriate control voltages for the thrusters, which provide the thrust forces to drive the ROV in accordance with the desired motions. The states of the vehicle are obtained by the sensors and filtered by a signal processing algorithm to eliminate random noise.These filtered signals are used for the next control iteration and the virtual reality monitor.

\subsection{Kalman filter for estimating ROV states}

The Kalman filter is an efficient recursive filter that estimates the state of a linear or nonlinear dynamic system from a series of noisy measurement [7]. In this project, the speeds and accelerationsof the ROV are acquired by the 6-DOF IMU sensor, with the signals including noise and bias. By fusing the estimated velocities with the data from sensor system, a Kalman filter reduces the variation of the noise, thus providing a 'cleaner' and improved feedback signal to the controller. In this paper, the surge, yaw rates and depth are estimated by using a Kalman filter.

\subsubsection{Estimation of surge, heave and yaw velocities}

The estimated model and observation equation are given by

$$
\dot{x}_{s}=A \cdot x_{s}+B(T)+w_{\text {noise }} ; \quad y_{s}=H \cdot x_{s}+z_{\text {sensor }}+v_{\text {noise }},
$$

where

$x_{s}$ : state vector which includes the surge, heave velocity and yaw rate $\mathrm{x}_{\mathrm{S}}=\left[\begin{array}{lll}u_{r} & v_{r} & r_{r}\end{array}\right]^{T}$;

$B(T)$ : torque matrix whose values are estimated in the section below;

$w_{\text {noise }}$ : process noise which is assumed to be a zero mean Gaussian white noise process with covariance matrix $\mathrm{Q}_{\text {noise }}=\left[\begin{array}{ccc}Q_{u} & 0 & 0 \\ 0 & Q_{w} & 0 \\ 0 & 0 & Q_{r}\end{array}\right]$;

$H$ : observer vector;

$z_{\text {sensor }}$ : state vector from the sensors;

$v_{\text {noise }}$ : noise vector whose covariance matrix is $R$.

Matrix $A$ in equations (6) can be derived from the kinetic equations of the ROV in equation (1) as follows

$A=M^{-1}[-C(v)-D(v)] ; B(T)=M^{-1} T$,

Step 1: Define the initial state vector and covariance matrix as

Covariance matrix of error $P(0)=E\left[\left(x_{s}(0)-\hat{x}_{s}(0)\right)\left(x_{s}(0)-\hat{x}_{s}(0)\right)^{T}\right]$.

Initial state vector

$$
\hat{x}_{\mathrm{S}}(0)=\left[\begin{array}{lll}
\hat{u}(0) & \hat{w}(0) & \hat{r}(0)
\end{array}\right]^{T} .
$$

Step 2: Calculate the Kalman gain as

$$
K(t)=\frac{P(t) \cdot H^{T}}{H \cdot P(t) \cdot H^{T}+R},
$$

with $\mathrm{K}(t)=\left[\begin{array}{ll}K_{1}(t) & K_{2}(t)\end{array}\right]$ 
Step 3: Estimate state propagation by updating the new state vector as per the following formula,

$$
\begin{aligned}
& \dot{\hat{x}}_{s}(t)=A \cdot \hat{x}_{s}(t)+B(T(t))+K(t)\left[y_{s}(t)-C \cdot \hat{x}_{s}(t)\right], \\
& \hat{x}_{\mathbf{s}}(t)=\hat{x}_{\mathbf{s}}(t)+\dot{\hat{x}}_{s}(t) d t .
\end{aligned}
$$

Step 4: Update error covariance matrix for the new iteration as

$$
\begin{aligned}
& \dot{P}(t)=A \cdot P(t)+P(t) \cdot A^{T}+Q-P(t) \cdot H^{T} \cdot R^{-1} \cdot H \cdot P(t), \\
& P(t)=P(t)+\dot{P}(t) d t .
\end{aligned}
$$

The loop is repeated from Step 2 until the end of the operating process.

\subsubsection{Estimation of the thrust force}

It can be seen that the thrust forces are required as input variables for the ROV state estimation algorithm. These parameters can not be measured directly, thus, in this section, an observer is proposed to estimate the thrust forces from the measurable variables.

From equation (2) the relationship between the input voltage $V_{a}$ and torque $Q$ is expressed as

$$
\frac{K_{t}}{L_{a} I} V_{a}=\ddot{\omega}+\left[\frac{R_{a}}{L_{a}}+\frac{b}{I}\right] \dot{\omega}+\left[\frac{R_{a} b}{L_{a} I}+\frac{K_{b} K_{t}}{L_{a} I}\right] \omega+\frac{1}{I} \dot{Q}+\frac{R_{a}}{L_{a} I} Q .
$$

To brief the above equation, the parameters are defined as

$$
b_{1}=\frac{K_{t}}{L_{a} I} V_{a} ; a_{1}=\left[\frac{R_{a} b}{L_{a} I}+\frac{K_{b} K_{t}}{L_{a} I}\right] ; a_{2}=\left[\frac{R_{a}}{L_{a}}+\frac{b}{I}\right] ; c_{1}=\frac{R_{a}}{L_{a} I} ; c_{2}=\frac{1}{I} .
$$

Replacing the newly defined parameters in Equation (15) yields the following formula

$$
b_{1} V_{a}=\ddot{\omega}+a_{2} \dot{\omega}+a_{1} \omega+c_{2} \dot{Q}+c_{1} Q .
$$

As mentioned, the parameters of a dc motor are known, except for the torque disturbances $\mathrm{Q}$ due to the environment. Thus, the observer is designed by

$$
b_{1} V_{a}=\ddot{\hat{\omega}}+a_{2} \dot{\hat{\omega}}+a_{1} \hat{\omega}+c_{2} \dot{\hat{Q}}+c_{1} \hat{Q}
$$

where $\hat{\omega}$ and $\hat{Q}$ are the rotational speed and estimated torque of the numerical actuator model respectively.

As shown in Section 3, the resistant torque $Q$ on the motor shaft has a linear relationship with the thrust force. Therefore, thrust $T$ is indirectly estimated by determining $Q$. For this purpose, the following rotational error between the two equations given in (17) and (18) must be minimised as follows. Accordingto Srissertpol [8], the following update rules are applied to satisfy the condition

$$
\frac{d^{2} \hat{Q}}{d t^{2}}=-\gamma_{2} \dot{e}_{\omega} c_{2} ; \quad \frac{d \hat{Q}}{d t}=-\gamma_{1} \dot{e}_{\omega} c_{1}
$$

Therefore, the formula representing the thrust force observation is presented as

$$
\dot{\hat{Q}}(t)=-\dot{e}_{\omega}(t)\left(\gamma_{1} c_{1}+\gamma_{2} c_{2}\right) ; \quad \hat{Q}(t)=\hat{Q}(t-1)+\dot{\hat{Q}}(t) .
$$

Substituting the estimated value of the resistant torque into Equation (5), the torque coefficient is calculated by

$$
\hat{K}_{Q}=\frac{\hat{Q}}{\rho D^{5} \omega|\omega|}
$$


From Equation (4), the advance ratio is obtained as

$$
\hat{J}_{o}=\frac{\hat{K}_{Q}-\beta_{2}}{\beta_{1}} .
$$

Substituting $\hat{J}_{0}$ into Equations $(4)$ and $(5)$, the estimated propeller thrust $(\hat{T})$ is derived.

\section{VIRTUAL REALITY SIMULATION}

\subsection{Virtual reality environment}

Based on the mathematical models that describe the relationships between the inputs and outputs, the VR system generates a similar performance to that of the physical system and provides the user with a realistic interaction experience. In this paper, a virtual ROV model is developed based on the hydrodynamic coefficients which are identified in Table 2. Based on the performances of the virtual model, the control system is evaluated and improved to meet the desired outcomes. A by-product is that a VR system is also a low cost operator training solution, which is based on easily accessible and relatively affordable equipment. Due to the ability to mimic the behaviour of the actual vehicle and its environment, VR provides operators with arealistic operating environment to prepare them for actual missions.
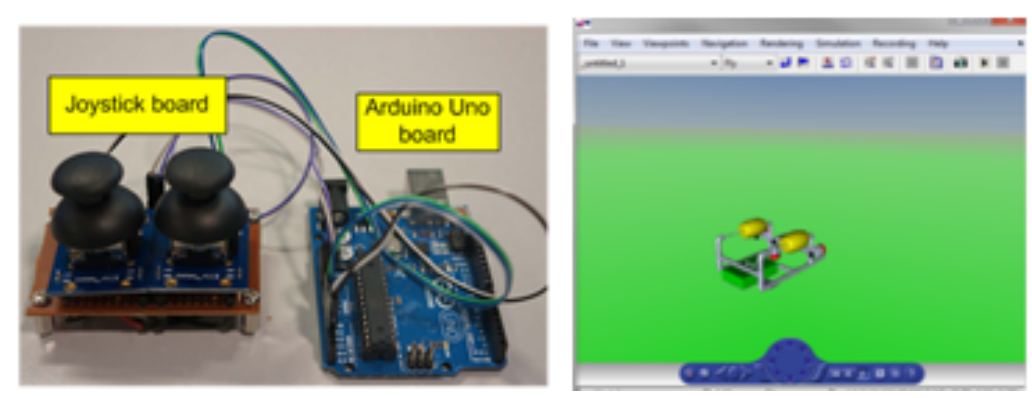

Figure 3. Control joystick and ROV model ina virtual environment

The dynamic model in Equation (3) and the parameters in Table 2 are used to develop the relationships between the input thruster forces and the output ROV speeds. The mathematical model of the thruster motors given in Equation (4) is applied and linked directly to the ROV dynamic model. In addition, an analogue joystick (Figure 3) using an Arduino controller was built and combined with the VR programme through a serial communication port.

\subsection{Simulation results}

The driving joystick system (Fig 3) was 'plugged' into and combined with the simulation programme. As the pilot pulls or pushes the button, the output voltage of the joystick is interpreted as the speed command and is used as the input for closed loop controller. For simplicity, the conventional PID control law is used to generate the control signal for the system by

$$
V_{a}=K_{p} e(t)+K_{i} \int e(t) d t+K_{d} \frac{\mathrm{d} e(t)}{d t},
$$


where $V_{a}$ is the control signal, $e(t)$ is the error, and $K_{p}, K_{i}$ and $K_{d}$ are the proportional, integral and derivative control gains, respectively. In this simulation, the PID gains are set as: $K_{p}=10, K_{i}=5$ and $K_{d}=0$. In order to test the robustness of the control system, a medium range water current is included by using the following random disturbance current profiles

$$
\frac{d \mathrm{v}_{c}}{d t}=-\mathrm{v}_{c}+B r_{n o i s e},
$$

where $r_{\text {noise }}$ is white noise, whose mean and variance are 0 and 0.2 , respectively, and $B$ is the parameter that identifies the range of the disturbance $(B=1)$.

In this study, an ROV operator attempts to control the ROV to follow the rectangular trajectories, which are predefined by the way points shown in Figure 4. It can be seen from the simulation results that the basic form of the desired path is achieved regardless of the effects from the external disturbance and sensor noise. The responses of the forward speed, yaw rate and depth are shown in Figure 5, from whose first three graphs it is seen that with the designed closed loop control system, the ROV is able to accurately follow the commands giving by the operator. The steady state errors between the reference values and the ROV responses are small (less than 5\%), and there is no significant overshoot at any point due to the high damping effect of the surrounding water.

There is the difference between the actual and the estimated forces due to the errors within the dc motor mathematical model. These errors lead to an unreliability of the results obtained from the ROV mathematical model.In addition, the raw data from the sensor also contain a white noise ( 0 mean and 0.05 variance) to simulate the performance of the actual sensors. However, by combining these two data sources, one from the ROV model and the other from the sensor system, the covariance of errors are significantly reduced with the effects of sensor noise mitigated so that the feedback data are smoother than the raw signal which is directly measured from the sensors (comparison of the red and the blue/dot lines in Figure 5). It shows that good feedback signals can be obtained by using Kalman filters, thus reducing the effect of noise and mathematical model uncertainty in order to improve the ROV performance.

From the three last graphs of Figure 5, it is seen that the under-actuated motions of the ROV are affected by the water current disturbances, centripetal force, Coriolis force, etc. The $2 \mathrm{D}$ trajectory graph shows that the water current disturbance in the sway direction distorts the path of the ROV. Since the number of actuators is less than the degrees of freedom, the ROV operator has to compensate for the path deviation by manipulating the heading angle of the ROV (yaw controller)in order to keep tracking the pre-defined way points.
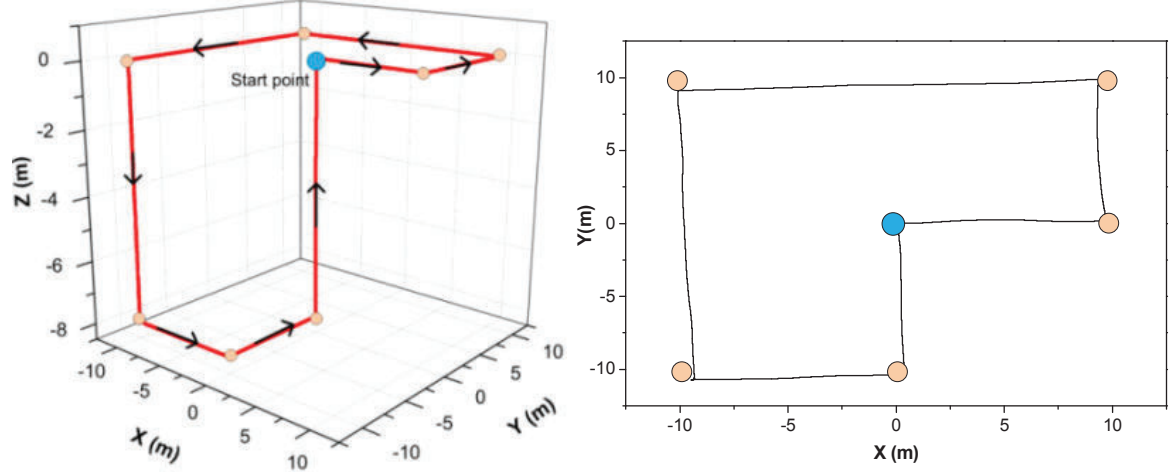

Figure 4. 3D and 2D trajectoryperformance of the ROV control by joystick 
The pitch and roll angles are caused by the coupling effect between the surge motion and centripetal force. These angles can not be controlled by the actuators, and are automatically restored to the equilibrium position by the righting moments created by the buoyancy and gravity forces. The pitch and roll fluctuation amplitudes dictate the stability characteristic of the vehicle, thus these angles must be kept as small as possible. The response of the pitch motion (Fig 5) shows that this angle is proportional to the forward speed. This is due to the thruster generating the moments as the vehicle moves forward, resulting in the pitch of the vehicle. In addition, the lift forces generated on the vehicle frame due to the forward motion contributes to the pitching moment. This is partially verified when noting that the pitching moment is a function of the forward speed. At thesurge speedof $0.5 \mathrm{~m} / \mathrm{s}$, the pitch and roll angles are small and the equilibrium positionis easily maintained by the restoring forces. Thus, the ROV system is stable and safe within the operating range speed.
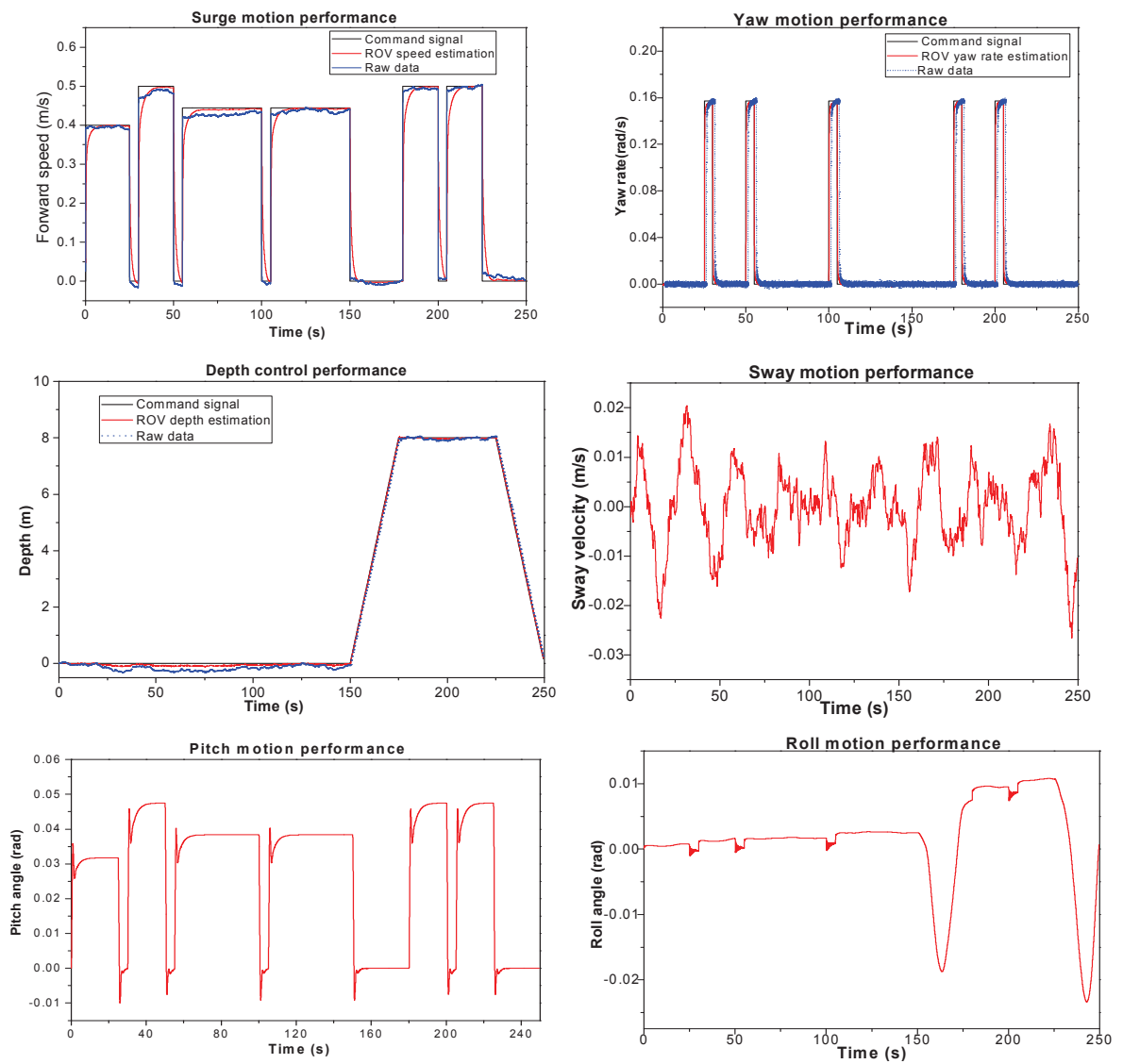

Figure 5. ROV responses

\section{CONCLUSIONS}

This paper has developed the hardware and the associated control algorithm for a low cost ROV. The open source Arduino electronic controller was utilised as the main on-board control unit. By connecting with various peripheral devices such as the IMU, pressure sensor, and motor drivers, the Arduino controller collected the relevant data from the ROV sensors 
and directed them to the onshore station computer. The data is processed by the advanced Kalman filter algorithm to reduce the influence of any external disturbances and noise, and then combined with the ROV operator's commands to generate the required control signals. In addition, a Virtual Reality (VR) model of ROV has been proposed to display the behaviour, spatial location, and attitude of the vehicle based on the feedback data. This effectively improves the controllability of the vehicle, as the operator can directly observe the performance of the ROV through different view-points and provide the required inputs to maintain the required trajectory. The simulations conducted are also able to validate the interaction between the operator using the thumb joystick and the response of the VR model. The closed-loop control system have been designed for surge rate, yaw rate and depth control. It facilitates the operating process by linearizing the relationship between the joystick signal and the ROV velocity. The simulation results have shown that the closed-loop system is stable in the operating scenario even with the existence of sensor noise and external environmental disturbances.

Further work is required to verify the performance of the vehicle by conducting free running model tests to evaluate the performance of the physical system under varying operational conditions. This will enable the VR model to be compared against the physical model for validation, thus providing a versatile design tool that can assist in the training of ROV operators.

\section{REFERENCES}

[1] D. A. Smallwood, L. L. Whitcomb, Model-based dynamic positioning of underwater robotic vehicles: theory and experiment, Oceanic Engineering, IEEE Journal of 29 (2004), 169186.

[2] A. R. Marzbanrad, M. Eghtesad, R. Kamali, A Robust Adaptive Fuzzy Sliding Mode Controller for Trajectory Tracking of ROVs, Decision and Control and European Control Conference (CDC-ECC), IEEE Conference on, Dec. 12-15, 2011, 2863-2870.

[3] T. Ken, E. An, P. P. J. Beaujean, A robust fuzzy autonomous underwater vehicle (auv) docking approach for unknown current disturbances, Oceanic Engineering, IEEE Journal of, $\mathbf{3 7}$ (2012), 143-155.

[4] H. D. Nguyen, S. Malalagama, D. Ranmuthugala, Design, modelling and simulation of a remotely operated vehicle-Part I, Journal of Computer Science and Cybernetics 29 (2013), 299-312.

[5] D. Gracanin, K. P. Valavanis, N. C. Tsourveloudis, M. Matijasevic, Virtual-environment-based navigation and control of underwater vehicles, Robotics $\&$ Automation Magazine, IEEE 6 (1999), 53-63.

[6] Q. Lin, C. Kuo, A virtual environment-based system for the navigation of underwater robots, Virtual Reality 3 (1998), 267-277.

[7] T. I. Fossen, Handbook of Marine Craft Hydrodynamics and Motion Control, John Wiley \& Son, United Kingdom, 2011.

[8] J. Srisertpol, C. Khajorntraidet, Estimation of DC motor variable torque using adaptive compensation Control and Decision Conference (CCDC '09), Chinese, 2009, 712-717.

Received on October 08, 2013

Revised on April 18, 2014 